\title{
Robot Social-Aware Navigation Framework to Accompany People walking Side-by-Side
}

\author{
Gonzalo Ferrer* • AnaísGarrell* · Fernando Herrero • Alberto Sanfeliu \\ *indicates equal contributions
}

Received: date / Accepted: date

\begin{abstract}
We present a novel robot social-aware navigation framework to walk side-by-side with people in crowded urban areas in a safety and natural way. The new system includes the following key issues: to propose a new robot social-aware navigation model to accompany a person; to extend the Social Force Model, "Extended Social-Force Model" (ESFM), to consider the person and robots interactions; to use a human predictor to estimate the destination of the person the robot is walking with; and to interactively learning the parameters of the social-aware navigation model using multimodal human feedback. Finally, a quantitative metric based on people's personal spaces and comfortableness criteria, is introduced in order to evaluate quantitatively the performance of the robot's task. The validation of the model is accomplished throughout an extensive set of simulations and real-life experiments. In addition, a volunteers' survey is used to measure the acceptability of our robot companion's behavior.
\end{abstract}

Keywords Robot Companion · Service Robots · Urban Robot Navigation · Human-Robot Interaction

\section{Introduction}

Nowadays, robots interact naturally with people and their environment, thus, urban robots require some skills to

G. Ferrer*, A. Garrell*, F. Herrero and A. Sanfeliu Institut de Robotica i Informatica, CSIC-UPC.

Llorens Artigas 4-6, 08028 Barcelona, Spain.

Tel.: +34934010863

Fax: +34 934015750

E-mail: \{gferrer,agarrell,fherrero,sanfeliu\}@iri.upc.edu This research was conducted at the Institut de Robòtica i Informàtica Industrial (CSIC-UPC). It was partially supported by CICYT projects DPI2007-61452 and Ingenio Consolider CSD2007-018 successfully serve people and being useful to them. Concretely, the walking side-by-side task is an important tool that any urban robot should have, and it responds to the basic necessity of accompany people in a safety and natural way; see Fig. 1. In this work, we define the walking side-by-side task in the context of service robots as an enhancement to the follow task where the robot does not know which is the persons destination, and needs to somehow infer it, to be able to walk sideby-side with him/her.

Robot companion is a multidisciplinary field of robotics in which intervenes a mixture of subjects such as perception, robot navigation and human robot interaction. Despite the heterogeneity of the subjects treated, the problem can not be tackled independently but in a holistic way, which is not an easy endeavor. Fig. 2 shows the components required for robot companion and where this work has made new contributions.

In the present work, we have extended the traditional way of guiding people to accompany people on a safety and natural way, taken into account that the robot does not know the accompany person destination. This new system includes new important features for the accompany task: predict where the person wants to go; predict other persons trajectories; and navigate in a socialaware manner in a typical human environment avoiding future collisions to the robot or the accompanied person. Moreover, the new system learns the best required parameters to do this task. The new social-aware navigation model is an extension of the Social Force Model (Helbing and Molnár, 1995), where the interactions between people and the robot are included.

To refine the overall system, additional considerations are required, such as performance function and a learning stage. On the one hand, we have developed a new metric to evaluate the robot's performance, based 


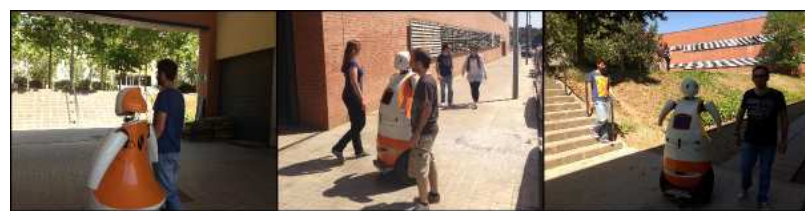

Fig. 1 Tibi accompanies a person. Tibi is walking side-byside with a pedestrian in an urban area in different scenarios.

on vital spaces and comfortableness criteria. On the other hand, we have proposed an interactive learning model to enhance the social-aware navigation using the human feedback response in the system.

The model was validated through a large set of simulations and real-life experiments, as well as a questionnaire administered to each individual who participated in the experiments.

In the remainder of the paper we start by discussing the related work in Section II. Section III makes an overview of the method. Section IV extends the theory of the Social-Force Model in a new Extended Social-Force model (ESFM) to describe human-robot interactions in terms of motion. Section V presents the human motion prediction. Section VI proposes the robot's novel socialaware navigation and the novel metric to evaluate the performance to accompany a person. Section VI also describes how human feedback is used in the interactive learning of the social-aware navigation parameters. The experimental results and conclusions are presented in sections VII and VIII, respectively.

\section{Related Work}

Lastly, human-robot interaction has become a very active research field, unfortunately, the research on motion planning in the presence of humans is still new in comparison to other fields. Some methods have been developed to allow robots to navigate around people while performing specific tasks. Some of these tasks include tending toward the right side of a hallway (Olivera and Simmons, 2002) and standing in line (Nakauchi and Simmons, 2002). Museum tour guide robots are often given the capability to detect and attempt to deal with people who are blocking their paths on a case-by-case basis (Burgard et al, 1999).

Several groups have begun to address questions relating to plan complete paths around people, rather than relying on solely reactive behaviors. In (Shi et al, 2008), a method for a robot to change its velocity near people has been developed. While this method begins to address ideas of planning around people, it does not directly consider social conventions. In contrast, in (Sisbot et al, 2007), the Human-Aware Motion Planner considers both the safety and reliability of the robots movement and human comfort, it attempts to keep the robot in front of people and visible at all times. However, the paths that the planner generates may be very unnatural due to its attempts to stay visible to people. In (Svenstrupet al, 2010) a trajectory planning algorithm for a robot navigation in dynamic human environments was introduced. Moreover, a new approach is proposed in (Stein et al, 2014), where the robot takes advantage of the motion of pedestrians to improve its navigation in dynamic environments. An experimental study was presented in (Vasquez et al, 2014), where the authors describe a comparison of Inverse Reinforcement Learning for socially compliant robot navigation in crowded environments.

In most works, unlike ours, safety is assured by not allowing humans to approach robots. However, these methods cannot be used if the robot has to assist a human. In (Alami et al, 2006), the notion of safety is studied in detail with all of its aspects in Human Robot Interaction.

Two different aspects of human's safety have been studied in (Zinn et al, 2004): "physical" safety and "mental" safety. With this distinction, the notion of safety includes physical aspects and psychological effects of the robots motions on humans. Physical safety is necessary for the human-robot interaction. Normally, physical safety is assured by avoiding collisions with humans and by minimizing the intensity of the impact in case of a collision.

Introducing the science of "proxemics", Hall demonstrates how man's use of space can affect personal business relations, cross-cultural exchanges, city planning and urban renewal (Hall , 1969). A robot should comply to similar conventions (Fong et al, 2003). In human robot interaction, the spatial formation around a robot has been studied in relation to initiating interaction (Michalowski et al, 2006). A classification of people's motion towards a robot was presented in (Bergstrom et al, 2008). In (Tasaki et al, 2004), a robot that chooses a target person based on distance was developed. A system for navigation in a hallway was described in (Pacchierotti et al, 2006), here, the proxemics rules are used to define the different strategies of interaction. Furthermore, the work in (Rios-Martinez et al, 2014) introduce a good review from sociological concepts to social robotics and human-aware navigation in terms of proxemics.

In (Madhava Krishna et al, 2006) a new approach to deal with safety and comfort issues is presented. In this research, the robot adapts its trajectory and its speed in order to guarantee that no collision will occur in a dynamic environment. Although the human is not consid- 
ered explicitly, this method guarantees a motion without collision by taking into account the dynamics of the environment.

Human-robot interaction research in the field of robots navigation side-by-side is still new in comparison to traditional service robotics, such as robots serving food in hospitals or providing specific security services. Therefore, prior research in this particular field is relatively minimal (Wilkes et al, 1997). In (Pandey and Alami, 2009) the authors presented a system for a mobile autonomous robot guide, which gives the opportunity to people to decide the manner they want to be guided.

Moreover, in HRI field the development of autonomous robots (Dautenhahn et al, 2005) to accompany people is an area of increasing interest. Thus, researchers are making efforts on performing human-robot interaction in a more natural way. A robot should detect the human operator and conduct his/her commands (Haasch et al, 2004).

In the majority of the current research studies, the robots participate in social-human interactions as companions (Ishiguro et al, 2001). In (Dautenhahn et al, 2006), the authors shown that a seated person prefers to be approached by a robot in a fetch-and-carry task from the front left or right direction rather than frontally or from behind. Further research showed that there are other mediating factors such as a persons experience with robots (Koay et al, 2007), gender (Dautenhahn et al, 2006) or in which part of the room she was standing or sitting (Walters et al, 2007). Satake et al. (Satake et al, 2009) proposed an approach model for robots, which should initiate interaction in a shopping mall.

In (Pransky, 2004), a new perspective to the different uses and identities of a companion robot has been brought, moreover it also describes the advantages and disadvantages of this type of companion. The "Robotic Butler/Maid" was able to perform domestic task, but also caused difficulties in relationships at home by being too efficient and making people feel redundant. In (Dautenhahn et al, 2005), a human-centered approach was adopted in order to look into people's perceptions and their desires for a companion robot. If social robots are going to be used in office and domestic environments, where they will have to interact with different individuals, they will have to be able to survive and perform tasks in dynamic, unpredictable environments and they must act safely and efficiently. The presence of human beings creates new problems for motion planning and control, as their security and comfort must be considered. The principal goal of the motion planner is to take human movements into account in order to ensure their safety.
In contrast, in this work we present a novel robot approach accompanying him/her to a predicted goal, walking side-by-side. We believe that most of the limitations of state-of-the-art methods are overcome by our method, as will be discussed in the following sections.

\section{Overview of the Method}

This article describes a new robot social-aware navigation framework to walk side-by-side with people in crowded urban areas, where the robot is considered as a social agent moving naturally in human environments accordingly to the Extended Social-Force Model (ESFM), and thus, aiming to a destination and reacting to obstacles and people. The navigation framework includes learning the basic parameters of the ESFM for the accompany functionality in order to adapt to human's movements and in this way, be accepted by people.

The contributions and topics described in the paper are presented below, see Fig. 2.

ESFM-based Robot Companion Social-Aware Navigation: A robot model capable of approaching a person and accompanying him/her to a predicted goal is presented. The social force model described in (Helbing and Molnár, 1995) takes into account both destinations and interactions by defining a summation of existing forces determining people trajectories. The meaning of "social force model" does not refer to a social robot's behavior, but rather to the existence of a non-physical force that robots can exert to move or drag people. More specifically, this work proposes a robot's reactive navigation based on an extension of the social force model which results from the internal motivations of the individuals performing certain movements (Zanlungo et al, 2011; Garrell and Sanfeliu, 2012).

Intentionality Prediction: We use an indicator capable of quantifying the human motion intentionality (HMI) implicit on a trajectory with respect to the current position and orientation. This intentionality indicator captures the probability that a human trajectory reaches a destination point. This prediction is used by the robot to predict the destination of the person who is being accompanied.

Interactive Robot Companion Parameters Learning: An interactive approach tests the model forces learned. At the same time, it learns which robot behavior is desired by humans. The provided feedback is a subjective measure, nevertheless, its purpose is to learn a general approaching rule that defines a better robot behavior. The proposed interactive learning helps to enlighten the nature of the model, in addition to generate controlled interaction forces that otherwise would be extremely complicated to generate. The on-line feedback 


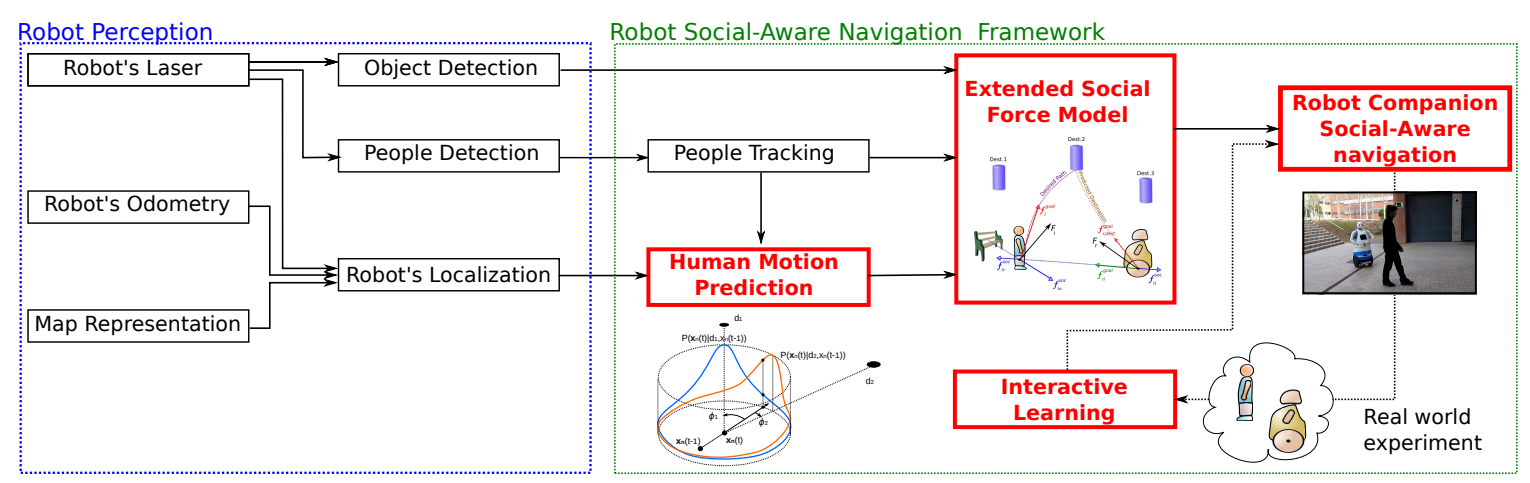

Fig. 2 Overview of the presented work. A general diagram of the robot framework is depicted, as well as its requirements. We have highlighted in red our contributions to advance the topics discussed later in the present work.

comes from the target person to whom the robot tries to approach.

Robot Companion Performance Metric: In order to evaluate the performance of the task accomplished by the robot, a quantitative metric is defined. This assessment is based on "proxemics", proposed by Hall (1969).

Other implemented issues we considered are: (i) Robot localization, it is mandatory for the deployment of robots in real-life environments. Accordingly, we are using a state of the art localization, the AMCL, which is a probabilistic localization system for a robot moving in 2D (Thrun et al, 2005)). (ii) People detection, to detect people, we implemented a laser detector based on (Arras et al, 2007). Finally, (iii) people tracking, we implemented the approach presented in (Luber et al, 2011b). According to our experience, the most critical source of error in the perception system occurs when the current track is lost due to long occlusions or lack of detections for geometrical reasons and a new id is assigned to the target. In these rare situations (but prone to eventually occur), a manual reassignment of the accompany target is required.

\section{Extended Social Force Model}

Human motion has been studied in many works (Helbing and Molnár, 1995; Zanlungo et al, 2011; Huang et al, 2006), where behavioral changes have been used in terms of social fields or forces. However, most of these models do not consider the interaction between people and robots. In order to achieve a model capable of representing the interactions between pedestrians, obstacles, and robots in typical social environments, we propose the Extended Social Force model (ESFM), based on (Helbing and Molnár, 1995). This new model expands the classical social force model including the force $\mathbf{F}_{i}^{\text {int }}$, which takes into account the interaction forces of people, $\mathbf{f}_{i, j}^{i n t}$, the interaction forces of obstacles, $\mathbf{f}_{i, o}^{\text {int }}$, and the

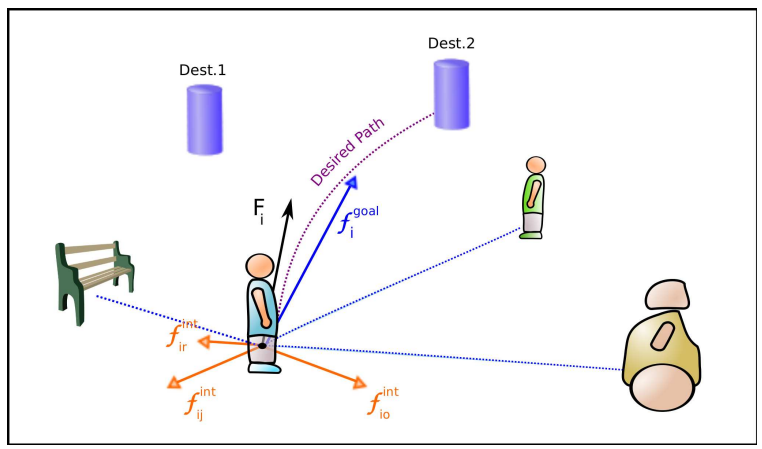

Fig. 3 Social-Force Model. Diagram of the social forces corresponding to the person $p_{i}$. The blue arrow represents the force aiming to a destination and the orange arrows represent each of the different kinds of interaction forces: personperson, object-person and robot-person. The summation of all the forces is represented as the black arrow $F_{i}$.

forces of the robot, $\mathbf{f}_{i, r}^{\text {int }}$. We will explain these forces in this section.

Formally, the ESFM considers the pedestrian $p_{i}$ with mass $m_{i}$ as a particle abiding the laws of Newtonian mechanics, and makes use of attractors and repulsors in the continuous space to characterize motion. Hence, the equation of motion for a pedestrian is given by the social force term

$\frac{d \boldsymbol{v}_{i}(t)}{d t} m_{i}=\boldsymbol{F}_{i}(t)$,

which describes the trajectory of the pedestrian $p_{i}$ over time. For the sake of simplicity, we will value $m_{i}$ as the unity for all considered people.

A person is attracted to a goal in the scene (as depicted in Fig. 3) through the steering force, $\boldsymbol{f}_{i}^{\text {goal }}$, but he/she is also influenced by other pedestrians $p_{j}, f_{i, j}^{i n t}$, by obstacles, $f_{i, o}^{i n t}$ and, in the present study we model the robot interaction $\boldsymbol{f}_{i, r}^{i n t}$. The resulting force $\boldsymbol{F}_{i}$ governs the trajectory described by the target $p_{i}$ is 


$$
\boldsymbol{F}_{i}=\boldsymbol{f}_{i}^{\text {goal }}+\boldsymbol{F}_{i}^{\text {int }}
$$

The attractive force $f_{i}^{\text {goal }}$ is defined by:

$$
\boldsymbol{f}_{i}^{\text {goal }}=k_{i}\left(\boldsymbol{v}_{i}^{0}-\boldsymbol{v}_{i}\right)
$$

where the pedestrian $p_{i}$ tries to move at a desired speed $v_{i}^{0}$ in a desired direction $\boldsymbol{e}_{i}$, such as $\boldsymbol{v}_{i}^{0}=v_{i}^{0} \boldsymbol{e}_{i}$. The desired velocity's direction is given by a vector pointing from the present position of the person $\boldsymbol{r}_{i}$ to the next subgoal $\boldsymbol{g}_{i}$, where the speed is the one at which the human feels more comfortable to walk. The relaxation time is the time needed to reach the desired velocity and the desired direction, where $k_{i}=1 / \tau_{i}$, and $\tau_{i}$ is the time for a human to take a step, 0.5 seconds approximately.

Furthermore, repulsive effects from the influences of other people, obstacles and robot in the environment are described by an interaction force $F_{i}^{i n t}$.

This force prevents humans from walking along their intended direction, thus, it is modeled as a summation of forces either introduced by people $p_{i}$, by static obstacles in the environment $o$ and the robot $r$. Fig. 3 shows a diagram of the social forces corresponding to the person $p_{i}$. The final formula of the ESFM is:

$$
\boldsymbol{F}_{i}^{i n t}=\sum_{j \in P} f_{i, j}^{i n t}+\sum_{o \in O} f_{i, o}^{i n t}+f_{i, r}^{i n t}
$$

where, $P$ is the set of people moving in the environment where the human interacts and $O$ is the set of obstacles. These forces are modeled as:

$$
\boldsymbol{f}_{i, q}^{i n t}=A_{q} e^{\left(d_{q}-d_{i, q}\right) / B_{q}} \frac{\boldsymbol{d}_{i, q}}{d_{i, q}}
$$

where $q \in P \cup O \cup\{r\}$ is either a person, an object of the environment or the robot. $A_{q}$ and $B_{q}$ denote respectively the strength and range of interaction force, $d_{q}$ is the sum of the radii of a pedestrian and an entity, and $\boldsymbol{d}_{i, q} \equiv \boldsymbol{r}_{i}-\boldsymbol{r}_{q}$. In order to calculate the Euclidean distance between $p_{i}$ and the entity $q$, humans and objects are assumed to be of circular shape with radii $r_{i}$ and $r_{q}$. The parameters $A_{q}, B_{q}, d_{q}$ are defined depending on the nature of the object. In this paper we obtain the parameters describing the robot-person interaction since, to the authors' knowledge, these parameters had not been obtained before.

Given the limited field of view of humans, influences might not be isotropic. This is formally expressed by scaling the interaction forces with an anisotropic factor depending on $\varphi_{p, q}$ between $\boldsymbol{v}_{i}$ and $\boldsymbol{d}_{i, q}$
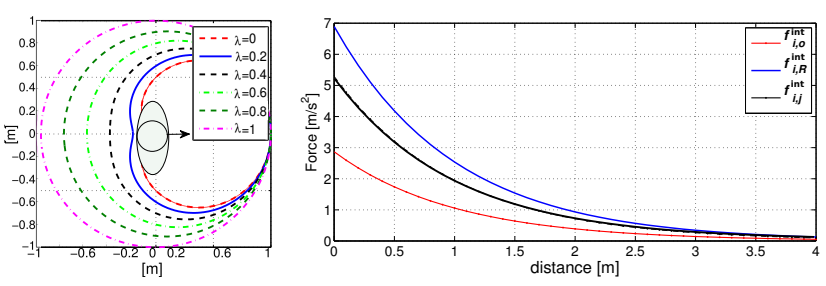

Fig. 4 Forces graph. Left: Representation of eq. 6, defined as the given limited field of view of human. Right: Forces magnitudes, the $\mathrm{x}$-axis shows the distance from person $p_{i}$ to an object, a person $p_{j}$ or the robot $r$. The radius of $p_{i}$ is $0.2 m$ and the sum of the radii of $p_{i}$ and $p_{k}$ is $0.4 \mathrm{~m}$. For a robot, the considered radius is $1 \mathrm{~m}$.

$w\left(\varphi_{i, q}\right)=\left(\lambda+(1-\lambda) \frac{1+\cos \left(\varphi_{i, q}\right)}{2}\right)$,

where $\lambda$ defines the strength of the anisotropic factor

$\cos \left(\varphi_{i, q}\right)=-\boldsymbol{n}_{i, q} \cdot \boldsymbol{e}_{r}$

The term $\boldsymbol{n}_{i, q}$ is the normalized vector pointing from q to person $p_{i}$ which describes the direction of the force. Fig. 4 presents the value of the presented forces and the anisotropic factor.

Finally, the described forces are used to define robot's motion, and thus, we combine with this pedestrian $d y$ namics model with a people tracker in order to obtain a more realistic human motion prediction.

\subsection{ESFM's parameters Learning}

As it was pointed previously, we considered three kinds of interaction forces: person-person, person-obstacle and person-robot. The first and the second interactions have been studied in previous papers like (Helbing and Molnár, 1995; Zanlungo et al, 2011; Luber et al, 2011a). However, the person-robot interaction parameters were not directly obtained in any previous work, thereby, in this section we present a learning method to obtain the parameters $\left\{k, A_{r p}, B_{r p}, \lambda_{r p}, d_{r p}\right\}$.

We decouple the training in two steps: First, we optimize the intrinsic parameters value of the model forces $\{k\}$, by describing the expected human trajectories under no external constrains. Second, we compute the optimal values of the extrinsic parameters of the force interaction model $\{A, B, \lambda, d\}$ under the presence of a moving robot, making sure it is the only external force altering the outcome of the described trajectory. These optimizations are carried out using genetic optimization algorithms (Goldberg, 1988) minimizing the 
following error function throughout all $N$ training trajectories:

$$
\{A, B, \lambda, d\}=\arg \min _{\{A, B, \lambda, d\}}\left\{\sum_{N} \sum_{\text {time }}\left\|\mathbf{x}_{\mathbf{o}}(\mathbf{t})-\mathbf{x}_{\mathbf{e}}(\mathbf{t})\right\|\right\}
$$

where $\mathbf{x}_{\mathbf{o}}$ is the person's observed position and $\mathbf{x}_{\mathbf{e}}$ is the value expected according to Eq. 1.

We developed a database in a real environment, as explained in Sec. 7.1.3, where we recorded more than 80 human trajectories using a laser sensor, and then, we manually labeled people's positions along the trajectory. A total of 20 volunteers participated in both parts of the experiment.

\section{Intentionality Prediction}

In the presented framework it is required a model capable of forecast the set of trajectories that any person might describe at any time. There have been developed different methods. (Bennewitz et al, 2005) proposed a learning-based prediction method, which learns basic patterns of human motion trajectories. (Ziebart et al, 2009; Henry et al, 2010; Kuderer et al, 2012) used the Inverse Reinforcement Learning (IRL) method to acquire human-like trajectories.

On the other hand, some works have made use of geometry criteria to obtain the final goal that a person aims to (Foka and Trahanias, 2010; Ferrer and Sanfeliu, 2011; Ikeda et al, 2012). In (Luber et al, 2011b) they have considered final destinations as linear propagations of the current state, but it only solves locally the problem whereas the long-term prediction remains unanswered.

The Extended Social Force Model requires the destination of the accompany person, that is, a long-term prediction method. Accordingly, we define a set of destination positions $D=\left\{d_{1}, d_{2}, \ldots, d_{M}\right\}$, that represents the human motion intentionality, like typically visited spots on an environment.

We are using the Bayesian Human Motion Intentionality Predictor (BHMIP) (Ferrer and Sanfeliu, 2014), a geometrical prediction indicator capable of identify the human motion intentionality on a trajectory with respect to the current position and orientation. The BHMIP captures the probability that a human trajectory reaches a destination point $d_{m}$, which is a clear indicator for the inherent intentionality.

To achieve this, we define the variable $\phi_{n m}$, which is the angle between the current orientation of the target $n$ and the vector to the destination point $d_{m}$, a relative measure of the orientation with respect to a destination (see Fig. 5 for clarification).

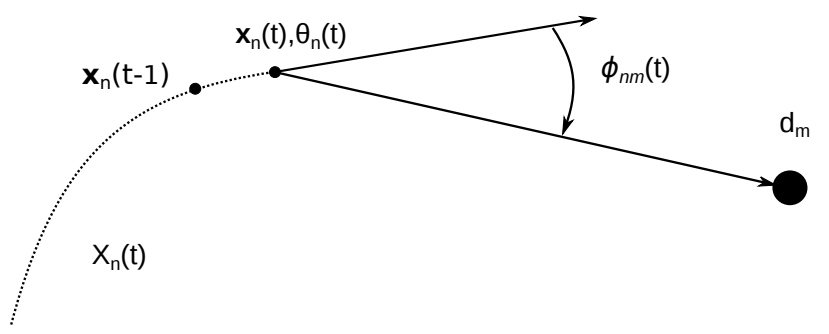

Fig. 5 Intentionality indicator. The angle $\phi_{n m}$ is defined as the angle described by the orientation vector of the target $n$ at time $t$ and the $\mathbf{x}_{\mathbf{n}}(t) \rightarrow d_{m}$ vector.

Briefly, we present a basic formulation necessary to analyze real trajectories. Let

$X_{n}(t)=\left\{\mathbf{x}_{\mathbf{n}}(1), \mathbf{x}_{\mathbf{n}}(2), \ldots, \mathbf{x}_{\mathbf{n}}(t)\right\}$

be a set of $T$ points (laser people detections) where each point $\mathbf{x}_{\mathbf{n}}(t)=[x(t), y(t)]_{n}$ is the position at time $t$ of the $n$th trajectory with respect to the world reference frame. Additionally, we define the orientation $\theta(t)_{n}$ as a function of the current position and the previous position, with respect to a global reference frame.

As it can be seen in Fig. $5, \phi_{n m}(t)$ is the angle defined by the first derivative of the current trajectory and the $\mathbf{x}_{\mathbf{n}}(t) \rightarrow d_{m}$ vector. By doing this, $\phi_{n m}(t)$ becomes a measure relative to a destination, while $\theta(t)$ is a global measure of the target orientation. This difference will allow us to obtain a good characterization of the human motion intentionality.

The problem of estimating the best destination is reduced to a sequential data classification, where the decision of choosing a destination is taken at each instant of time while the human is walking. Our technique is inspired by a complete Bayesian framework, in order to classify the motion intentionality.

The BHMIP is a geometric-based prediction method which uses a Naive Bayesian classifier to compute the best prediction to a given destination position $d_{m}$, for each position $\mathbf{x}_{\mathbf{n}}(t)$ of the trajectory $X_{n}=\left\{\mathbf{x}_{\mathbf{n}}(1)\right.$, $\left.\mathbf{x}_{\mathbf{n}}(2), \ldots, \mathbf{x}_{\mathbf{n}}(t)\right\}$. We model the probability

$P\left(\mathbf{x}_{\mathbf{n}}(t) \mid \mathbf{x}_{\mathbf{n}}(t-1), d_{m}\right)=\mathcal{N}\left(\phi ; 0, \sigma_{\phi}^{2}\right)$

as a Gaussian function. In Fig. 6 is depicted an example of this probability function to two destinations centered at the position $\mathbf{x}_{\mathbf{n}}(t)$.

Using the Bayes theorem we can compute the posterior probability that the destination $d_{m}$, given the current and previous positions of the trajectory $X_{n}(t)$ : 


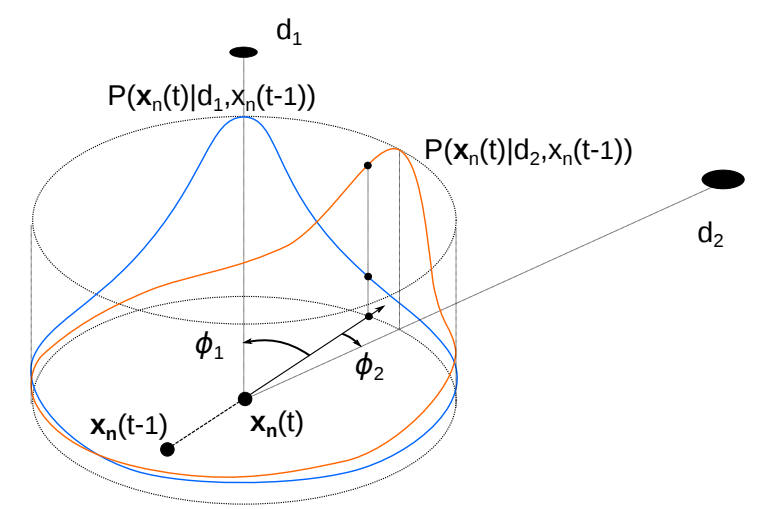

Fig. 6 Probability functions. Different probability functions shifted depending on their respective destinations.

$P\left(d_{m} \mid X_{n}(t)\right)=\frac{P\left(X_{n}(t) \mid d_{m}\right) P\left(d_{m}\right)}{P\left(X_{n}(t)\right)}$,

where $P\left(d_{m}\right)$ is the prior probability to reach the destination $d_{m}$ and the conditional probability $P\left(X_{n}(t) \mid d_{m}\right)$ can be formulated as:

$P\left(X_{n}(t) \mid d_{m}\right)=P\left(\mathbf{x}_{\mathbf{n}}(1) \mid d_{m}\right) \prod_{\tau=2}^{t} P\left(\mathbf{x}_{\mathbf{n}}(\tau) \mid d_{m}, \mathbf{x}_{\mathbf{n}}(\tau-1)\right)$

By replacing Eq. (12) into the Eq. (11), we can obtain a compact formulation of the BHMIP. For a more detailed discussion of the BHMIP intentionality predictor and the validation of the model, see (Ferrer and Sanfeliu, 2014).

\section{Social-Aware Robot Companion}

We present a novel approach called the social-aware navigation, driven by the ESFM centered at the robot, similar to the work by (Khatib, 1985), but stressing on the social nature of the approach. This navigation model makes use of the ESFM framework to successfully accompany a person while safely navigating in a crowded environment, avoiding either static and dynamical objects.

The mission of the robot is to navigate accompanying the person on a safety and natural way, which implies that the social-aware robot companion has to deal with two goals at the same time. Meanwhile the robot navigates towards the person predicted destination, at the same time, the robot has to approach the person who accompanies. Then two forces appears: the first force drives the robot towards the predicted destination $\mathbf{f}_{r, \text { dest }}^{\text {goal }}$ and the second force pushes the robot to move closer to the person $p_{i}, \mathbf{f}_{r, i}^{\text {goal }}$. The trade off of these forces, in addition to the interacting forces, describes the resultant force governing the robot movement:

$$
\boldsymbol{F}^{r}=\alpha \boldsymbol{f}_{r, \text { dest }}^{\text {goal }}+\beta \boldsymbol{f}_{r, i}^{\text {goal }}+\gamma \boldsymbol{F}_{r}^{\text {per }}+\delta \boldsymbol{F}_{r}^{o b s}
$$

Section 6.3 discusses the procedure to obtain the value of the parameters $\{\alpha, \beta, \gamma, \delta\}$ and how to update them.

This equation computes the force that the robot has to apply for navigating and is computed at each interval of time.

Let us going to describe all the forces intervening in the navigation approach. The force to the target's destination is inferred by using the intentionality prediction described in section 5, and thus the robot aims to the target's most expectable destination. Similarly occurs for the force to the accompanied person, trying to reach the position $\mathbf{x}_{i}$ :

$$
\begin{aligned}
& \boldsymbol{f}_{r, \text { dest }}^{\text {goal }}=k_{r}\left(\boldsymbol{v}_{r}^{0}\left(P\left(d_{m} \mid X_{i}\right)\right)-\boldsymbol{v}_{r}\right) \\
& \left.\boldsymbol{f}_{r, i}^{\text {goal }}=k_{r}\left(\boldsymbol{v}_{r}^{0}\left(\boldsymbol{x}_{i}\right)\right)-\boldsymbol{v}_{r}\right) .
\end{aligned}
$$

The force of interaction due to pedestrians is the sum of all the repulsive forces that each person generates to the robot, and is computed as follows:

$\boldsymbol{F}_{r}^{p e r}=\sum_{j \in P} \boldsymbol{f}_{r, j}^{i n t}$

where the force $\boldsymbol{f}_{r, j}^{i n t}$, which represents the interaction between the pedestrian $j$ and the robot, is computed as follows:

$$
\boldsymbol{f}_{r, j}^{i n t}=A_{r p} e^{\left(d_{r p}-d_{r, j}\right) / B_{r p}} w\left(\varphi_{r, j}, \lambda_{r p}\right)
$$

This is the formulation of the spherical force (Eq. 5) using the parameters $\left\{A_{p r}, B_{p r}, \lambda_{p r}, d_{p r}\right\}$. These parameters correspond to the person-to-robot interaction, and in general are dependent of the robotic platform.

Correspondingly, the interaction between robot and obstacles is computed as:

$\boldsymbol{F}_{r}^{o b s}=\sum_{o \in O} \boldsymbol{f}_{r, o}^{i n t}$

where $f_{r, o}^{i n t}$ is obtained as follows

$\boldsymbol{f}_{r, o}^{i n t}=A_{r o} e^{\left(d_{r o}-d_{r, o}\right) / B_{r o}} w\left(\varphi_{r, o}, \lambda_{r o}\right)$ 


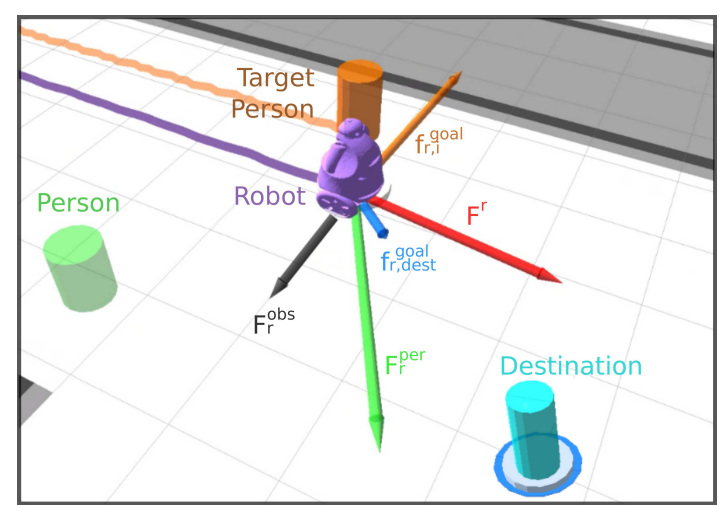

Fig. 7 Robot's Social Forces: Forces applied to the robot while accompanies a person.

It uses the specific parameters $\left\{A_{\text {ro }}, B_{\text {ro }}, \lambda_{\text {ro }}, d_{\text {ro }}\right\}$ corresponding to the interaction person-obstacle.

As it can be seen in Fig. 7, we have defined an additional destination to the robot approach. The robot aims to the target person in order to accompany him/her, following the Eq. 15.

Similarly as presented in section 4 , repulsive effects from the influences of other people and obstacles in the environment are described by an interaction force, which is a sum of forces either introduced by people or by static obstacles in the environment.

Additionally, other constraints have to be taken into account. All those robot's propagations which result in a collision with an obstacle, are forbidden. Current robot maximum velocity is also a constraint, it depends on the robot navigation state, which is a function of the proximity of persons. These velocity restrictions are shown in the following formula:

$v=\left\{\begin{array}{l}v_{\text {safety }} \text { if } \frac{d_{r, p}}{w\left(\varphi_{r, p}\right)} \leq \mu_{\text {safety }} \\ v_{\text {cruise }} \text { if } \mu_{\text {safety }}<\frac{d_{r, p}}{w\left(\varphi_{r, p}\right)} \leq \mu_{\text {social }} \\ v_{\text {free }} \text { otherwise }\end{array}\right.$

The $v_{\text {safety }}$ is the maximum velocity the robot can achieve when at least one person is inside its inner safety zone. We have proposed a social distance to define this region as $d_{r, p} w\left(\varphi_{r, p}\right)$, similarly as described in Sec. 4 , as a metric of the relative distance between the robot and a pedestrian and an asymmetric factor deforming the distance measure $w\left(\varphi_{r, p}\right)$. This condition also corresponds to the inner robot navigation state. On the other hand, $v_{\text {cruise }}$ is the cruise velocity when someone is inside its social safety zone and $v_{\text {free }}$ is the maximum robot velocity when there are no people inside its safety zone. The navigation states associated to this configurations are the social robot navigation and the free robot navigation, correspondingly.

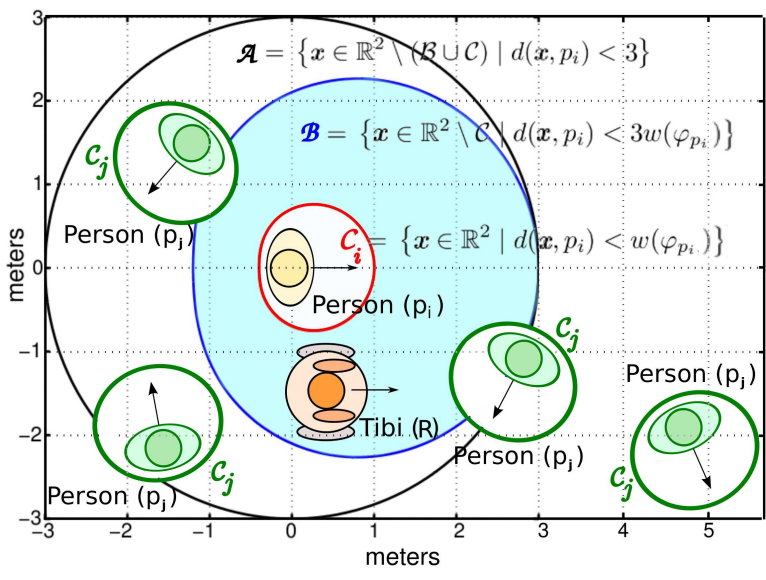

Fig. 8 Quantitative Metrics: Diagram of the areas used in the evaluation of the robot's performance. The areas represented by $\mathcal{C}_{j}$ are people's personal space, where the robot cannot get in, area named as $\mathcal{B}$ is the optimal area where the robot should be allocated in order to accompany a person. Finally, area $\mathcal{A}$ represents a social acceptable distance, but its performance is lower since if the robot is behind the person it would be out of the field of vision.

Prior values of the velocity parameters are obtained from the work presented in (Trulls et al, 2011), moreover, during the experimentation process, we redefine them according to our experience. Intuitively, these values are similar to pedestrians' velocity in urban areas when normally walking. If the robot lags behind (due to obstacles etc.), the $v_{\text {free }}$, which is the highest velocity value of all three proposed, it will set the robot into the desired B zone. If the target walks too "fast" to be caught, then the robot can't afford caught him/her without compromising safety. Furthermore, even if the robot would navigate at high velocities in the free zone, once someone enters in its social zone, then the maximum velocity becomes $v_{\text {cruise }}$ and even in the case someone gets even nearer the robot, there is a third maximum value, the $v_{\text {safety }}$, which is even smaller. By doing this, we minimize the risk of collision with other pedestrians and minimize the perception of thread in the proximity of other people.

\subsection{Quantitative Metrics}

To evaluate the performance of the task accomplished by the robot, a quantitative metric is defined. This assessment is based on "proxemics", proposed by Hall (1969) and the importance to walk side-by-side. This work considers the following taxonomy of distances between people:

- Intimate distance: the presence of another person is unmistakable, close friends or lovers $(0-45 \mathrm{~cm})$. 
- Personal distance: comfortable spacing, friends $(45 \mathrm{~cm}-$ $1.22 \mathrm{~m})$.

- Social distance: limited involvement, non-friends interaction $(1.22 \mathrm{~m}-3 \mathrm{~m})$.

- Public distance: outside circle of involvement, public speaking $(>3 \mathrm{~m})$.

To define the metric used in the present work, four different areas must be defined: (i) Personal space $\mathcal{C}_{i}$ of pedestrian $p_{i}$, robot's navigation has to be socially accepted by the person being accompanied, it is necessary that the robot does not perturb the human's vital space and walk beside the person, eq. 21. (ii) Social distance area $\mathcal{A}$, robots must be allocated in an acceptance social distance. (iii) The robot should be in the human's field of view as they interact during the performance of the task and must walk beside the person $\mathcal{B}$. (iv) Finally, there are other pedestrians in the environment $p_{j}$, the robot is not allowed to perturb pedestrians' personal space $\bigcup_{p_{j}} \mathcal{C}_{j}$.

$$
\begin{aligned}
& \mathcal{A}=\left\{x \in \mathbb{R}^{2} \backslash(\mathcal{B} \cup \mathcal{C}) \mid d\left(x, p_{i}\right)<3\right\} \\
& \mathcal{B}=\left\{x \in \mathbb{R}^{2} \backslash \mathcal{C} \mid d\left(x, p_{i} \pm\right)<3 w\left(\varphi_{p_{i}}\right)\right\} \\
& \mathcal{G}=\left\{x \in \mathbb{R}^{2} \mid d\left(x, p_{i}\right)<w\left(\varphi_{p_{i}}\right)\right\}
\end{aligned}
$$

where $w\left(\varphi_{p_{i}}\right)$ is defined in eq. 6 .

Moreover, the robot has beeen represented as a circle of 1 meter of diameter, with center robot's position $p_{r}, \mathcal{R}=\left\{x \in \mathbb{R}^{2} \mid d(x, r)<0.5\right\}$, whose area is $|\mathcal{R}|=\frac{\pi}{4}$.

Thus, we can now define the performance of the task accomplished by the robot, depending on human's position $p_{i}$ and robot's position $p_{r}$.

$\rho\left(r, p_{i}\right)=\int_{\left(\mathcal{B} \backslash \bigcup_{p_{j}} \mathcal{C}_{j}\right) \cap \mathcal{R}} \frac{d \mathbf{x}}{|\mathcal{R}|}+\int_{\left(\mathcal{A} \backslash \bigcup_{p_{j}} \mathcal{C}_{j}\right) \cap \mathcal{R}} \frac{d \mathbf{x}}{2|\mathcal{R}|} \in[0,1]$

Where $\mathbf{x} \in \mathbb{R}^{2}$. The range of the performance function is defined between 0 and 1 . If the complete area of the robot is allocated in zone $\mathcal{B}$, the performance is maxim, i.e., 1. As the robot moves far from the person and enters to zone $\mathcal{A}$, the performance decreases to 0.5. Finally, the performance in zones $\mathcal{C}_{i}$ is 0 , as it is not allowed that the robot enters in people's personal space.

\subsection{Parameter Learning}

To learn the values of the parameters $\theta=\{\alpha, \beta, \gamma, \delta\}$, we use a two-step learning approach. First, we require

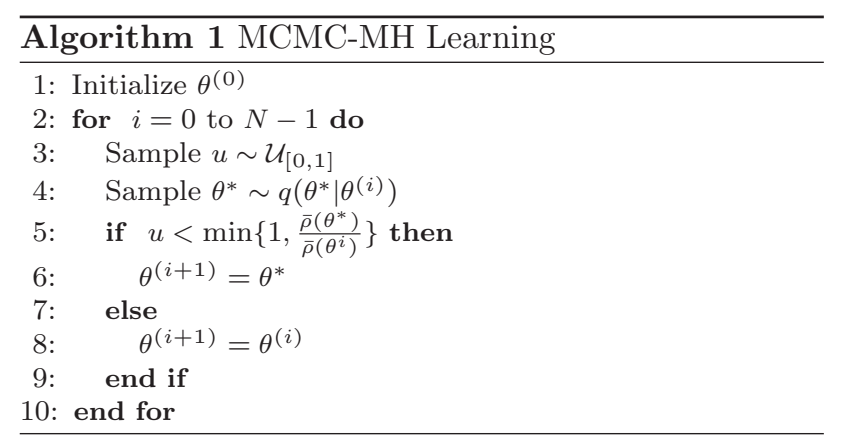

an initial estimation to learn the magnitude of the $\theta$ parameters.

An important issue when determining volunteers preferences in Human-Robot Interaction is the interpretation of the subjects' responses during the trials (Koay et al, 2006a). Consequently, it would be wise to define the overall performance of the task accomplished by the robot:

$\bar{\rho}(\theta)=\frac{1}{T} \sum_{t=0}^{T} \rho\left(p_{r}, p_{i}\right)$,

where $p_{i}$ is the interacting human's position, $p_{r}$ is the robot's position, and $\rho\left(p_{r}, p_{i}\right)$ is the metric function introduced in Section 6.1.

The initial conditions can be identically from the simulations, nevertheless, given the interactive nature of the approach, the parameters $\theta$ alter the outcome $\bar{\rho}(\theta)$ for each experiment (random variable). That is the main reason for considering stochastic optimization as an appropriate method for estimating the navigation parameters. Monte Carlo methods (Andrieu et al, 2003) are especially useful for simulating phenomena with significant uncertainty in inputs and systems with a large number of coupled degrees of freedom. More concretely, we have implemented a Markov Chain Monte Carlo Metropolis-Hastings (MCMC-MH) algorithm to find the best set of $\theta$, implementing the Alg. 1.

The term $q\left(\theta^{*} \mid \theta^{(i)}\right)$, appearing in the algorithm description, represents a Gaussian sampling for each parameter, centered at $\theta^{(i)}$ and a given variance for each of the random variables, which are considered as independent.

Then, we obtain the best $\hat{\theta}$ parameters as follows:

$\hat{\theta}=\arg \max _{\theta}\left\{E_{P(\theta)}\left\{\sum_{t} \bar{\rho}(t, \theta)\right\}\right\}$.

Note that the outcome of the simulations is averaged using the expectation $E_{P(\theta)}\{\}$ over the probability function of $\theta$. 


\subsection{Interactive Learning}

The second step of the learning approach consist of an Interactive Learning (Katagami and Yamada, 2003). Our approach requires a people's feedback response to refine the parameters values $\{\alpha, \beta, \gamma\}$. This method helps to enlighten the nature of the model, in addition, to generate controlled interaction forces that would otherwise be extremely complicated to generate.

The on-line feedback comes from the target person. The human agent provides the interaction by using a wii remote control. Here, the feedback measure is received from the subjective comfortableness of the target being approached.

This feedback is a subjective measure, nevertheless, that we have modeled weighting the contribution of all active forces. Volunteers had a wii remote control. Participants were told to press the button ' + ' if they wanted the robot to get closer to them. However, if people preferred the robot to move directly to the destination, they should push button '-'. Below, the variation of parameters depending on people's feedback is presented.

Firstly, we can define the function $N(T)$ as follows:

$$
N(T)=\sum_{t=0}^{T} \epsilon(t)
$$

where $\epsilon(t)$ is expressed as:

$\epsilon(t)=\left\{\begin{array}{l}0 \text { if human does not press any button } \\ +1 \text { if human presses button ' }+ \text { ' at time } t \\ -1 \text { if human presses button '-' at time } t\end{array}\right.$

$N(T)$ is the difference between the number of times the person presses button '+' and button '-' at time $T$. Then, $N(T) \geq 0$. If $N(T)<0$ we impose $N(T)=0$.

Secondly, the forces that appear during the process of accompanying vary according to the distance between the robot and the person. Then, the variation of the parameters will change depending on such distance.

Formally, if $h(N(T))$ denotes the function corresponding to human's response, it can be expressed as:

$$
h(N(T))= \begin{cases}\alpha(N(T)), \beta(N(T)) & \text { if } d_{r, i} \geq w\left(\varphi_{r, i}\right) \\ \gamma(N(T)) & \text { if } d_{r, i}<w\left(\varphi_{r, i}\right)\end{cases}
$$

where, $\{\alpha(N(T)), \beta(N(T)), \gamma(N(T))\}$ is the set of weighting functions for the parameters $\{\alpha, \beta, \gamma\}, d_{r, i}$ is the distance between the robot and the person, and, $w\left(\varphi_{r, i}\right)$ represents the personal space of a person, see eq. 6 .
Below, we explain how to compute the parameters of the equation 13.

Force to the target destination $(\alpha)$ : We infer the target of the destination by using the intentionality prediction described in section 5 , and thus the robot aims to the target's destination that is most expectable. As it has been described above, a parameter $\alpha$ controls the magnitude of the force $\boldsymbol{f}_{r, \text { dest }}^{\text {goal }}$. The value of this parameter is computed as follows:

$\alpha(N(T))=\log \left(1+N(T) / \sigma_{\alpha}\right)$

Force to the person being accompanied ( $\boldsymbol{\beta})$ : Above, it has been described an attractive force towards the accompanied person. Either the current target position and the expected motion prediction are known. Thus, the parameter $\beta$ controls the magnitude of the force $\mathbf{f}_{r, i}^{\text {goal }}$. This value is computed as follows:

$\beta(N(T))=1-\alpha(N(T))$

Force of interaction with people $(\gamma)$ : It must be considered a repulsive force due to the relative position and velocity between the robot and people, $\sum_{j \in P} \mathbf{f}_{r, j}^{i n t}$, this force is controlled by the parameter $\gamma$. The value of $\gamma$ is defined as:

$\gamma(N(T))=\log \left(1+N(T) / \sigma_{\gamma}\right)$

Force of interaction with obstacles $(\delta)$ : Finally, a repulsive force due to the relative position and velocity between the robot and obstacles has to be considered, $\sum_{o \in O} f_{r, o}^{i n t}$, this force is controlled by the parameter $\delta$. This parameter is not refined with human feedback since it only involves robot and obstacles.

Finally, after a number of trials with different humans which give feedback to the robot, we will obtain the best weights where the people will feel comfortable under the presence of the robot. The weighted sum of these four forces will determine the robot behavior for navigating and doing the accompany task.

\section{Experiments}

In this section, we will present the corresponding experiments to evaluate the complete system. An overview of this section is depicted in Fig. 9, where it can observed the order in which the experiments have been carried out, and in some of the modules, their generated output.

We first start this section presenting the robot platform, the experiment environment and the volunteer 


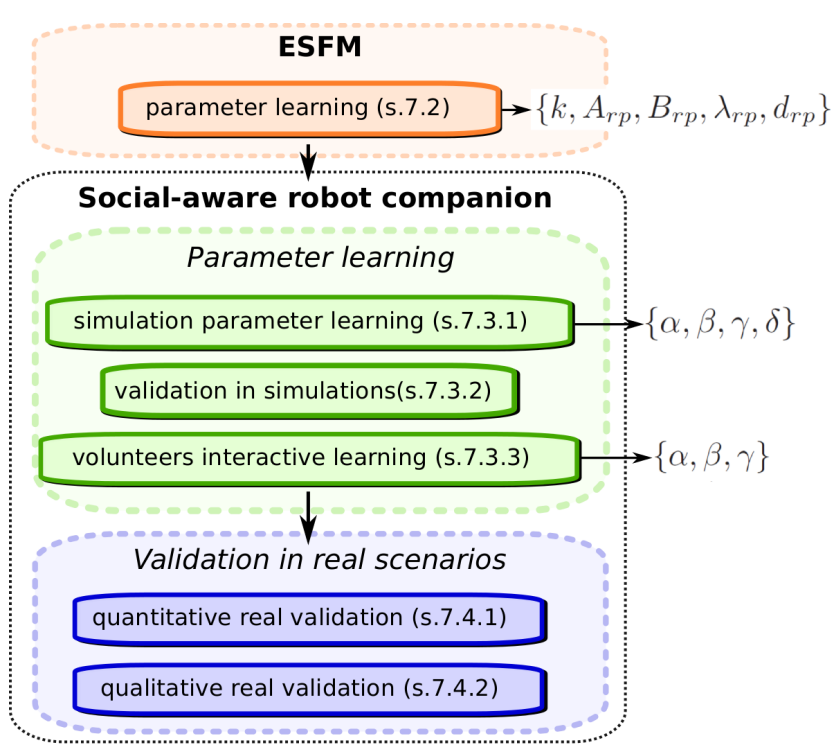

Fig. 9 Overview of the section: the sequence of experiments carried out, in decreasing order. In the right hand of the figure appears the output of some modules, for instance, the set of parameters learned.

profiles. In Sec. 7.2 we will describe the experiments carried out to obtain the ESFM parameters. In Sec. 7.3 we will present the simulation environment and how it has been used for computing and validating the parameters for the ESFM. In Sec. 7.3.3 we introduce how these obtained parameters are refined in a real scenario using the interactive learning method with volunteers. Finally, in Sec. 7.4 we will validate our approach in real scenarios, using as quantitative metric, the one described in Sec. 6.1, and as qualitative procedure, a volunteers questionnaire.

7.1 Robotic Platform, Testing Environment and Participants

\subsubsection{Robotic Platform}

To conduct all the real-life experiments and to test the approach presented, we have used two twin mobile service robots developed in the URUS project (Trulls et al, 2011), called Tibi and Dabo, designed to work in dynamic urban pedestrian areas and for interacting with people.

They are based on a two-wheeled Segway RMP200 platform, which works as an inverted pendulum in constant balancing, can rotate on the spot (nonholonomic), has wheel encoders providing odometry and inclinometers providing pitch and roll data.

To perceive the environment they are equipped with two Hokuyo UTM-30LX 2D laser range sensors used

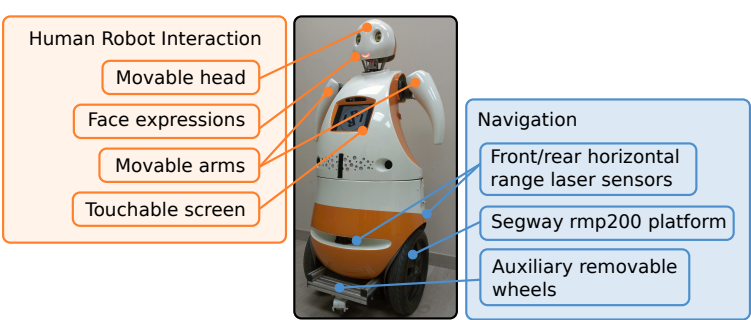

Fig. 10 Tibi Robot. Urban mobile robot used in the experiments.
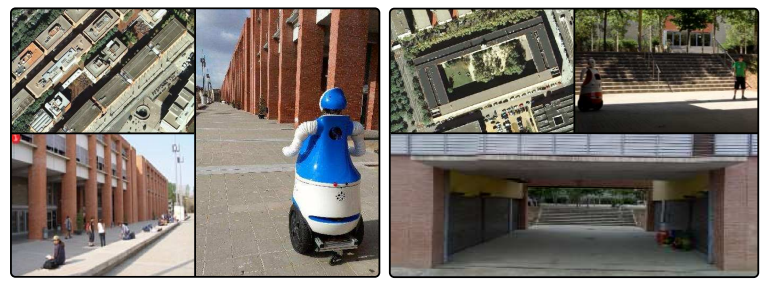

Fig. 11 Testing scenarios. Left: Barcelona Robot Lab, in the North campus University. Right: FME Lab, South campus of the University.

to detect obstacles and people, giving scans over a local horizontal plane at $40 \mathrm{~cm}$ above the ground, facing forward and backward. A stereo Bumblebee camera located in the eyes is used for computer vision purposes.

Tibi and Dabo are meant to interact with people as social robots. They have a set of interaction elements to perform human-robot-interaction, such as a touchable screen, speaker, movable arms and head, and illuminated LEDs for face expressions. Power is supplied by two sets of batteries, one for the Segway platform and one for the computers and sensors, giving about a $5 \mathrm{~h}$ full working autonomy. Two onboard computers (Intel Core 2 Quad CPU @ 2.66 and 3.00 GHz) manage all the running processes and sensor signals, and a laptop is used for external monitoring. The systems run Ubuntu-Linux and ROS as a middleware. The perception system runs at $20 \mathrm{~Hz}$ and the social-aware robot companion at $10 \mathrm{~Hz}$. This ensures that we can rapidly adapt to external changes in the environment and to sensing errors. Fig. 10 shows one of the robots and some of its components.

\subsubsection{Testing Environments}

The experimental areas where the experiments were conducted are the BRL (Barcelona Robot Lab) and the FME (Facultat de Matemàtiques i Estadística) lab, both outdoor urban environments located respectively at the North and South Campus of the Universitat Politècnica de Catalunya (UPC), 
The BRL (Fig. 11-Left) is a large section of the campus that was outfitted as an experimental area, covering over $10.000 \mathrm{~m}^{2}$, including six buildings and a square, with multiple ramps, staircases and typical obstacles such as bulletin boards, bicycle stands, trashcans or flower pots. The FME lab (Fig. 11-right) consists of a tree area and a pavement area separated each other by stairs.

\subsubsection{Experiments volunteers}

A total of 81 volunteers between 20 and $40(M=2 \% .4$, $S D=5.78)$ years old participated in the real experiments. None of them had experience working or interacting with robots, they represented a variety of university majors and occupations including computer science, mathematics, biology, finance and chemistry. We separated them into three different groups. First, a set of 20 persons participated in order to learn the ESFM parameters $k, A, B, \lambda, d$ and a second group of 20 volunteers for the $\alpha, \beta, \gamma$ parameters using the interactive learning. Finally, we evaluated the performance of the system considering a qualitative and quantitative analysis using the third group of 41 volunteers.

\subsection{ESFM parameter learning}

The first step for the robot approaching task is the study of the parameters of the ESFM. As it has been pointed above, we consider three kinds of interaction forces: person person, person-obstacle and person-robot. The first and the second interactions have been studied in previous papers (Helbing and Molnár, 1995; Zanlungo et al, 2011). However, the person-robot interaction parameters were not directly obtained in any previous work, thereby, in this section we present the results obtained for the parameters $\left\{A_{r p}, B_{r p}, \lambda_{r p}, d_{r p}\right\}$.

As discussed in Sec. 4.1, we have divided the parameter estimation in two different parts, analyzing 80 trajectories of different 20 volunteers. First, we optimize the intrinsic parameter $\{k\}$ describing the expected $h u$ man trajectories under no external constrains.

Second, we optimize the parameters of the force interaction model $\left\{A_{r p}, B_{r p}, \lambda_{r p}, d_{r p}\right\}$ under the presence of our moving robot

Table 1 shows the parameters learned after applying the minimization process (see Sec. 4.1), using genetic algorithms, to all database trajectories. Each parameter includes a standard deviation obtained after estimating the parameters for each trajectory independently. The standard deviation of some parameters is high, because people behave differently when they interact with robots. Furthermore, in this table, we have included the

\begin{tabular}{|l||c|c|c|c|c|}
\hline Int. & $\mathrm{k}\left[\mathrm{s}^{-1}\right]$ & $\mathrm{A}\left[\mathrm{m} / \mathrm{s}^{-1}\right]$ & $\mathrm{B}[\mathrm{m}]$ & $\mathrm{d}[\mathrm{m}]$ & $\lambda$ \\
\hline \hline Per-Per & 2 & 1.25 & 0.1 & 0.2 & 0.5 \\
\hline Per-Per & 4.9 & 10 & 0.34 & 0.16 & 1 \\
\hline $\begin{array}{l}\text { Rob-Per } \\
\text { (ours) }\end{array}$ & $\begin{array}{c}2.3 \\
( \pm 0.37)\end{array}$ & $\begin{array}{c}2.66 \\
( \pm 4.51)\end{array}$ & $\begin{array}{c}0.79 \\
( \pm 0.21)\end{array}$ & $\begin{array}{c}0.4 \\
( \pm 0.25)\end{array}$ & $\begin{array}{c}0.59 \\
( \pm 0.36)\end{array}$ \\
\hline
\end{tabular}

Table 1 Model forces parameters

parameters proposed by (Luber et al, 2010; Zanlungo et al, 2011) referred to the person-person SFM.

7.3 Social-aware robot companion. Parameter learning

We have devised different experiments to obtain the parameters that define the presented robot companion approach $\{\alpha, \beta, \gamma, \delta\}$. In this section we will describe the experiments for the two proposed learning methods: a simulated environment and a posterior refinement in a real scenario with some volunteers.

\subsubsection{Simulation environment for parameter learning}

To obtain a proper initial estimation of the $\theta=\{\alpha, \beta$, $\gamma, \delta\}$ parameters, and to evaluate mathematically the correctness of the navigation model, we have built a simulated dynamic environment. This simulated environment serves for two purposes: first, it allows an initial guess of the system parameters $\theta$ and, second, the simulated environment permits us to validate the performance of the approach, using the function defined in Sec. 6.1, in different scenarios and under different density of pedestrians.

To this end, we have implemented a complete social environment, depicted in the top row of Fig. 12. It takes into account pedestrians, obstacles and robots in an interactive way and each element is reactive to its surroundings, according to the ESFM. By doing this, we obtained a dynamical environment, in which each action of the robot alters the behavior of nearby pedestrians and vice versa.

According to previous human-based companion works (Garrell and Sanfeliu, 2012), we have set the accompany position at $1.5 \mathrm{~m}$ from the target and $60^{\circ}$ from the target's heading. Our approach for this task is evaluated in a simulated environment, which makes use of the social-aware robot companion and prediction information regarding the target destination. This simulated environment consists of a urban setting (inspired by the BRL), in which obstacles are present as well as a fixed number of pedestrians. 
To obtain a good initial estimation of the system parameters, we have carried out a large number of simulations, as explained above in Sec. 6.2, and we have run more than two thousand $M C M C-M H$ simulations. The outcome of each experiment is dependent on the parameters $\theta$, since the system reacts to the behavior of the robot navigation and vice versa. After applying the optimization method proposed in Sec. 6.2, we have obtained an initial guess of the magnitude of $\theta$ roughly rounded to the values $\{\alpha=0.1, \beta=0.6, \gamma=5.0$, $\delta=0.50\}$. It is important to note that these parameters are obtained after random initializations and in a virtual environment. However, this initial guess serves as a good estimation of $\theta$ and their corresponding standard deviations, which will be the initial values of the system parameters that we will use for the interactive learning.

\subsubsection{Validation in simulation of the prior parameters}

The second objective of the simulations is the validation of the parameters obtained before in a challenging environment. Our method makes use of the ESFM while approaching the target person, and additionally it uses prediction information regarding the target destination to enhance its performance (red in Fig. 12). We have also implemented two additional methods in order to compare them with our approach. A second configuration takes into account only the ESFM model (green in Fig. 12). Our method is also compared to a robot companion based on proxemics, where the robot follows the target person, not considering the force of interactions with other persons. When some person enters the robot inner safety zone, the robot stops until the path is clear (black lines in Fig. 12).

The experiment settings have been tested in three different scenarios, as can be seen in the top row of Fig. 12. The first setting is an unconstrained area, free of obstacles, where four destinations are defined. The second is a urban setting, in which obstacles are present as well as pedestrians. The third is a variation of the previous setting, but increasing the number of obstacles in the scene.

For each environment, the algorithms have been tested depending on the density of persons in the unoccupied area. To give statistical consistency to the results, more than 50,000 experiments have been carried out, only varying the initial conditions, which are the initial positions of each pedestrian in the scene and the destination they are aiming to. These conditions are calculated randomly and the robot has to accompany a person under this uncertain environment. We would like to stress on the fact that the environment has a high density of persons and each person aims to a random destination. This generates rapidly a chaotic and challenging environment for the robot companion testing (see video at the project web).

We consider our robot companion approach as a kind of potential navigation and despite of their already well known limitations (Khatib, 1985; Koren and Borenstein, 1991), in which local minima problems may appear, we did not experience such problems since either the destination as well as the person to be accompanied, were at all times describing affordable configurations of navigation.

The second row of Fig. 12 shows the overall performance of the different methods with respect to the density of pedestrians in the scene (Sec.6.1), taking into account penalizations due to nearby persons. As it is has been expected, using the social interaction forces (red and green lines) highly increases the performance, since the social-aware robot navigation helps to improve its efficiency. The predictive behavior clearly enhances the performance of the task, as it can be seen in the first and second columns. Under a reduce number of obstacles, the predictive information becomes very useful. However, under a high number of obstacles in the scene, the prediction strategy scores equally. This is due to the fact that acting predictively, specially in the third scenario, makes the robot to decide alternative paths rather than just follow the target; e.g. while moving, surrounding a column to the right when the target goes to the left, but the destination would be more accessible by doing this. Although this behavior denotes some intelligence, its performance is punished based on our metric, as can be seen in the figure. Nevertheless, the companion performance using prediction improves the overall performance of the system Fig. 12, third row.

The third row of Fig. 12 shows the average percentage of successful arrivals to the destinations, that is, if the robot is within the companion zone (zone A, see Sec. 6.1) at the moment the target achieves its destination. Although this metric is highly correlated, we can observe that using prediction increases the arrival rate with respect to the other methods. That is, the prediction information raises the probability to achieve its goal simultaneously to the target.

\subsubsection{Interactive Learning using volunteers}

In order to refine the value of the $\theta$ parameters, obtained in Sec. 7.3, it is required to test the system in a real environment (Fig. 11) with 20 different volunteers. The experiment setting uses a robot in a real scenario as follows: we explain to each volunteer to naturally walk towards its chosen destination, among two options rep- 

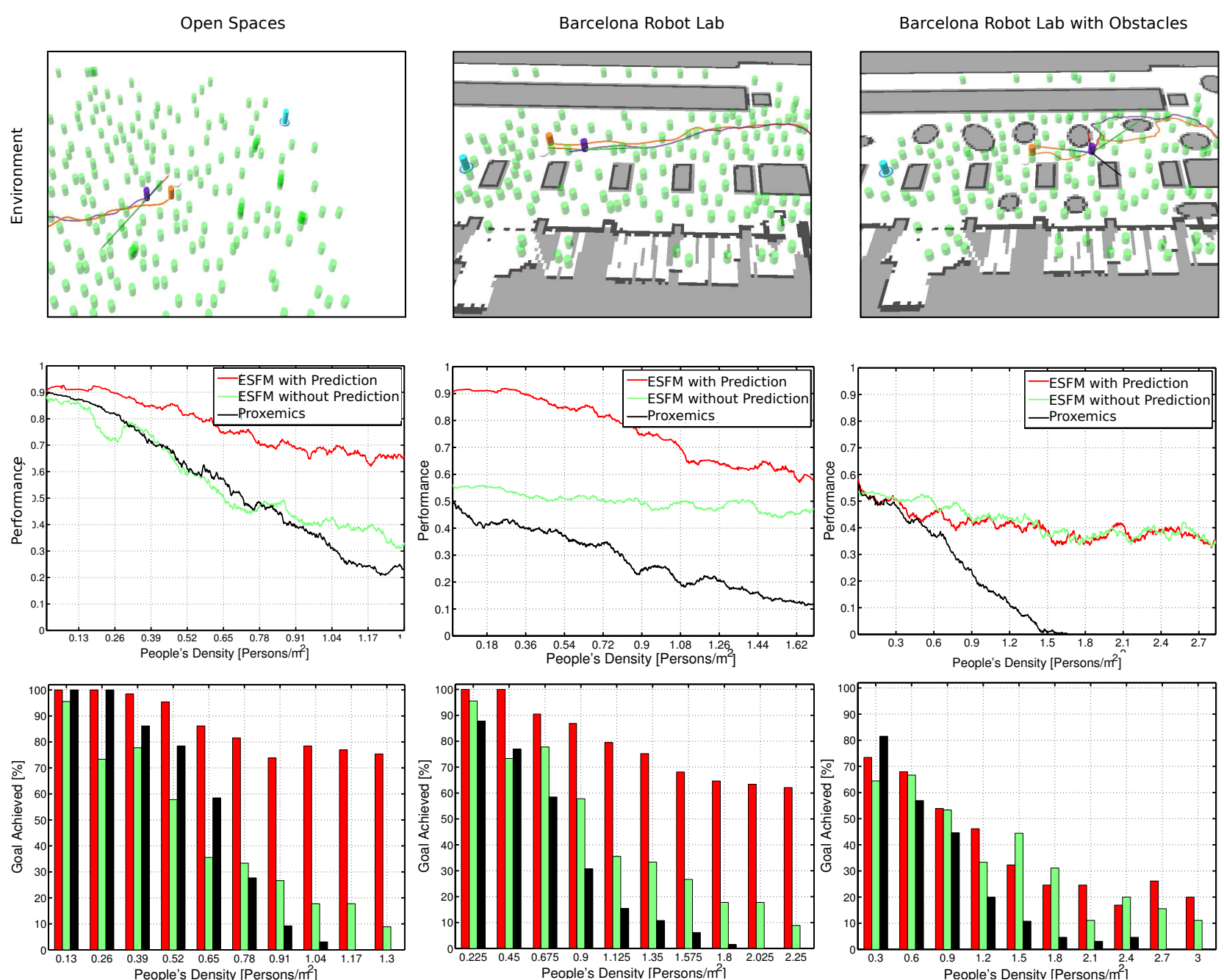

Fig. 12 Simulations comparative. Left column: unconstrained area with four destinations. Central column: urban settings, corresponding to the Barcelona Robot Lab. Right column: variation of the BRL with extra obstacles. Top row: different scenarios of the simulations, the blue cylinder corresponds to the destination, the orange one represents the person being accompanied by the robot and the purple one is the robot, the set of green cylinders are people in the environment. Second row: simulations performance, in black the proxemics approach, in green the ESFM companion and red the ESFM with prediction information. All results are function of the pedestrian density in the environment. Third row: rate of successful robot arrivals in bar diagrams. More info on the experiment videos in the project web (see Sec. 8).

resented as red pylons. While approaching the desired destination, the robot accompanies the volunteers and they should behave naturally. In addition, and stressing on the predictive nature of the approach, we set an intermediate goal, not learned by the robot, to represent disturbances in people's trajectories and observe how our approach deals with them.

As part of the interactive multimodal learning, the system learns the desired robot behavior as explained in Sec. 6.3. The feedback provided by the target is a subjective measure, nevertheless, its purpose is to learn a general approaching rule that defines a better robot behavior. It is provided directly by the target agent to be approached using a remote control, in this way the

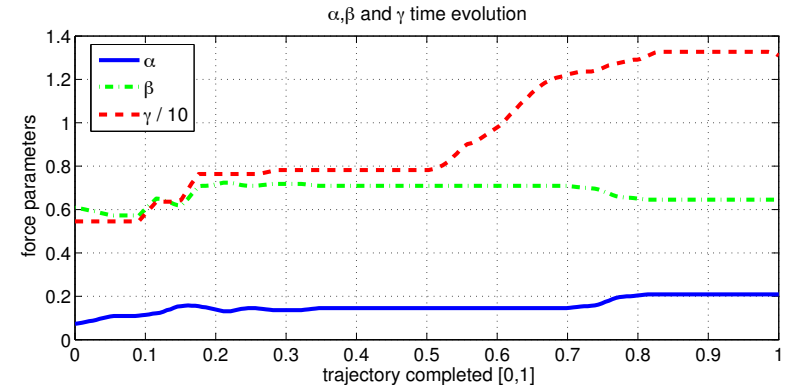

Fig. 13 Force parameters $\alpha, \beta, \gamma$. Evolution in time from start to end of each experiment of the force parameters $\alpha, \beta$, $\gamma$. These variables are averaged using results chosen for each participant during the experiment. 
system automatically weights the contribution of the active forces (Sec. 6.3). An initial estimation of the $\theta$ parameters is calculated in the simulations (sec. 7.3.1), and this information is useful although a readjustment is provided by the human feedback.

Fig. 13 shows the obtained $\{\alpha, \beta, \gamma\}$ weights from the user feedback that determines the robot behavior. It has been averaged using 20 different experiments and it is depicted as a function of time, normalized from the beginning to the ending of the experiment $t \in[0,1]$. The $\delta$ parameter is not recalculated using this method since no obstacles were present in the experiment.

\subsection{Robot companion validation in real scenarios}

Here, all the parameters of the system have been obtained and refined, as described previously. Consequently, we can evaluate the performance of the robot task approach in a real scenario, either using the quantitative metric explained in Sec. 6.1 or a qualitative method such a questionnaire to the volunteers. A total of 41 volunteers carried out the experiments. The results were analyzed using both the quantitative metric and a questionnaire that reveals a qualitative metric for the approach. We have prepared a set of four experiments combining prediction characteristics and feedback information in order to evaluate the overall performance of each combination:

- Without prediction and without feedback

- With prediction and without feedback

- Without prediction and with feedback

- With prediction and with feedback

We have kept the feedback in the system to probe if a direct control over the robot navigation parameters enhances the perception of people towards the system, specially for the questionnaires.

\subsubsection{Quantitative validation in real scenarios}

The robot was able to achieve its goal (the target's goal) in all the conducted experiments. The volunteers were told to naturally walk and the robot accompanied each person using the social-awareness navigation described in Sec. 6. During the validation of the model in the real experiments, we included unexpected obstacles and pedestrians in the targets path, and the robot avoided them successfully.

In Fig. 14 is depicted the average outcome of the performance during some experiments. Each group corresponds to a different configuration of the experiment settings. A summary of the overall performance for each setting is depicted in Fig. 15. The prediction information approach slightly enhances the robot companion approach. Below will be discussed again this comparison using the human poll results, and it will be showed clearly the advantages of using prediction information.

During the real-life experimentation appeared unexpected difficulties that were not taken into account during the simulations. We found limitations on the perception system, specifically in the people detector and tracker. People were not always detected and the data association somethings were wrong. However, a discussion of the perception system is out of the scope of the present paper and it is important to note that any other system (vision detection, RF, etc..) would also be subject to limitations under realistic conditions.

This new set of experiments, were performed mostly in the BRL, under the interaction of multiple pedestrians and obstacles. Fig. 16 depicts examples of different experiments performed with volunteers in different urban environments. Moreover, it is shown several case scenarios where multiple objects and people were in the scene. In Fig. 17, an example of a single sequence is depicted, where a robot accompanies a person under the presence of a group of people in the scene.

\subsubsection{Qualitative validation in real scenarios}

The component of the human responses has a great importance to measure the suitability of our robot companion (Koay et al, 2006b), for that reason, we conducted a survey to evaluate the robot behavior.

As it has been mentioned previously, 41 real-life experiments with different volunteers have been carried out. Each participant filled out a questionnaire. The measurement was a simple rating on a Likert scale between 1 to 7. For the evaluation score, repeated ANOVA measurements were conducted. In this section, we provide two different results. On the one hand, it is desired to know if the prediction skill increases the perception of robot's abilities on humans. And, on the other hand, it is necessary to study if the use of the remote control enhances the interaction between the robot and a person.

Firstly, human perception has been studied in the prediction skill. To analyze the source of the difference, three different scores were examined: "Robot's Intelligence", "Human-Like Motion" and "Level of confidence", plotted in Fig. 18. For robot's intelligence a repeated-measures analysis of variance revealed a significant main effect, $F(1,44)=14.82, p<0.001$. For robot's Human-like motion the ANOVA test revealed a great effect, $F(1,44)=36.28 p<0.001$. And, finally, 

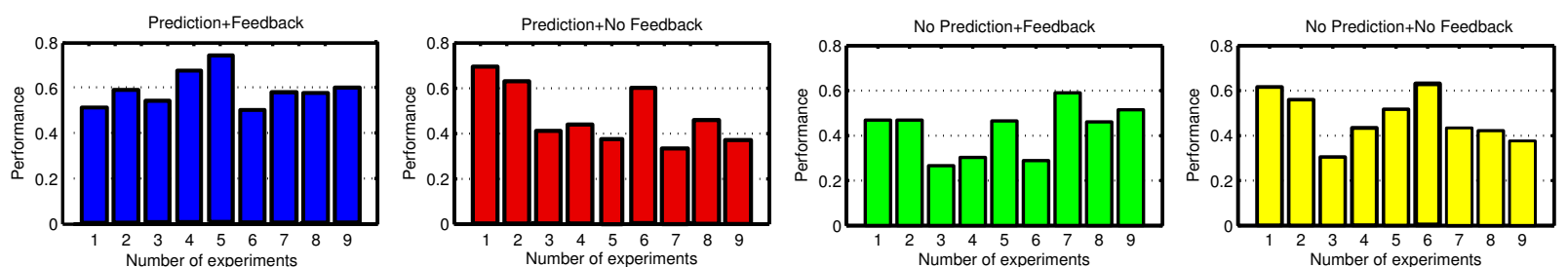

Fig. 14 Experiment results: A bar diagram describing the average performance of each of the real experiments carried out. Each color corresponds to a different experiment setting.

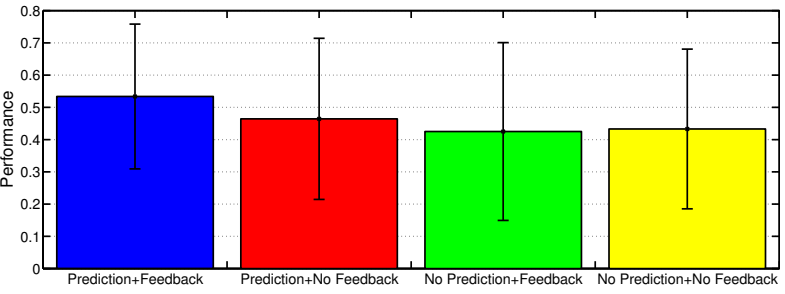

Fig. 15 Experiment results: An average of the performance and its standard deviation for the experiments combination using prediction and feedback.

the analysis of variance revealed a remarkable difference in the level of confidence $F(1,44)=61.79 p<0.001$.

Secondly, in order to analyze if the use of the remote control enhances the interaction between the robot and a person, three different scores were examined: "Robot's Intelligence", "Level of interaction" and "Level of confidence", plotted in Fig. 19. For robot's intelligence a repeated-measures analysis of variance revealed that no significance was found, $F(1,44)=1.88, p=0.18$. For the level of interaction the ANOVA test do not revealed a significant main effect, $F(1,44)=0.48 p=0.18$. And, finally, the analysis of variance showed that there is not a remarkable difference in the level of confidence $F(1$, 44) $=3.57 p=0.07$.

As a summary, after analyzing these two components, we may conclude that if the robot has the ability to predict human destinations, it has the largest acceptance. People perceived the robot to be more intelligent, as it could detect and approach them, and, they felt that it had more social skills. However, the use of the remote control does not enhance the interaction between the robot and a person.

We would like to point the reader to check the videos of the experimental results in the project web http:// www.iri.upc.edu/groups/lrobots/robot_companion/

\section{Conclusions and Future Work}

In this work, we have presented a new robot framework to accompany people based on the so called Social-Forces Model. Concretely, we have gone one step further extending the SFM, we included new robot-person inter- action force in our new model called Extended SocialForce model (ESFM). Moreover, we have developed a new social-aware navigation framework based on the social-forces concept and a human intentionality prediction model. This new system is well suited for a robot that walks side-by-side. A better performance has been demonstrated if human interactions are taken into account, specially in open spaces. To improve the performance of the robot-person interaction, we have learned the force parameters for the robots that perform the accompany tasks, in our case the Tibi and Dabo robots.

Moreover, a new robot metric has been defined. Since the verification of any system in which a human intervenes is hard to evaluate, we require an analytical metric to evaluate the behavior of our robot while accompany people.

Finally, we have introduced a model of human feedback that is able to obtain the set of weighting parameters for the robot behavior. We believe that human feedback for parameter learning is a key point for the development of robots whose purpose is interacting with people.

The validation of the model has been demonstrated throughout an extensive set of simulations and real-life experiments in a urban area. In contrast to other existing approaches, our method can handle realistic situations, such as dealing with large environments with obstacles and highly crowded scenes. For that reason, this work can be applied to certain specific real robot applications, for instance, guiding tourists or accompanying professional visitors. The overall validation of the approach in real scenarios is done by using feedback information directly from the volunteers.

Moreover, we can determine that introducing a human intentionality predictor greatly increases the overall human-perceived performance of the system, according to the surveys. Nevertheless, the multimodal feedback under the shape of a wii remote controller did not improved the subjective performance, according to the poll.

In future work, we aim to obtain more sophisticated robot behavior, by exploring the enhancement of the model of the human motion prediction. A temporal propagated model may increase even more the perfor- 


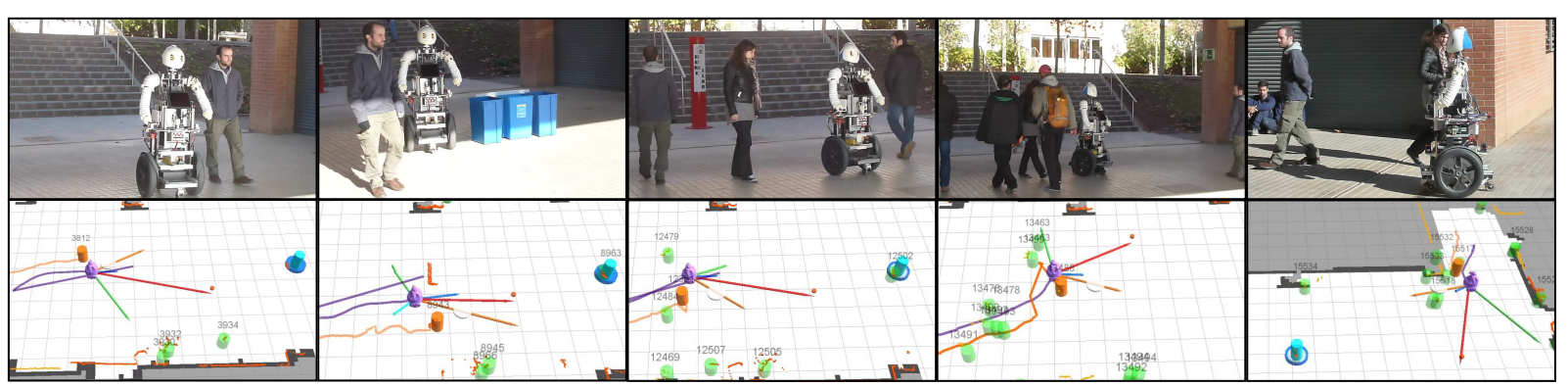

Fig. 16 Real-life experiments: Some examples of the conducted real experiments. Top: Dabo accompanying a person to a desired goal. Bottom: The same scene using the system interface.

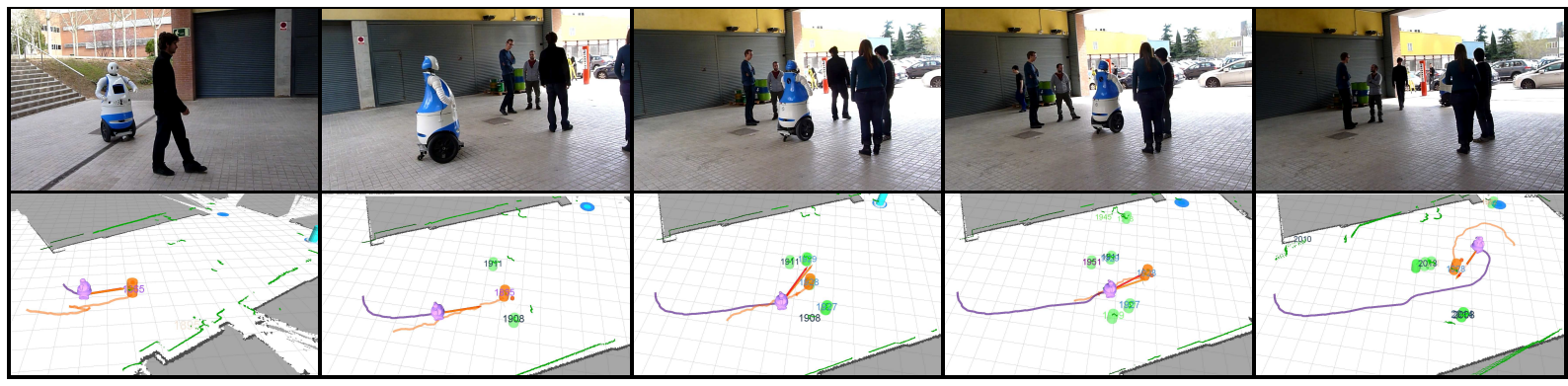

Fig. 17 Real-life experiments 2: A single robot companion experiment. Top: Dabo accompanying a person to a desired goal. Bottom: The same scene using the system interface.
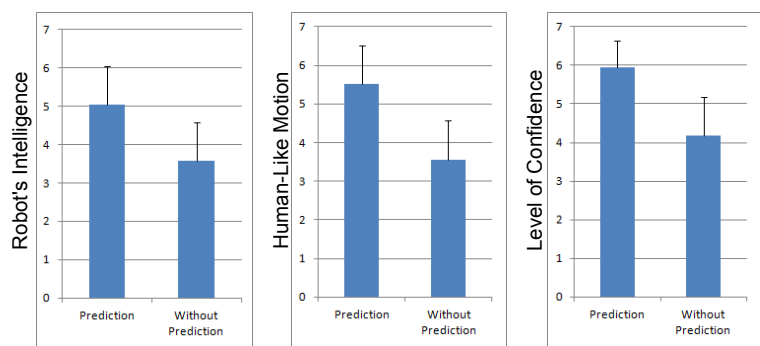

Fig. 18 Prediction skill. People's perception of the prediction skill. Left: Robot's Intelligence. Center: Robot's Humanlike motion. Right: Level of confidence.
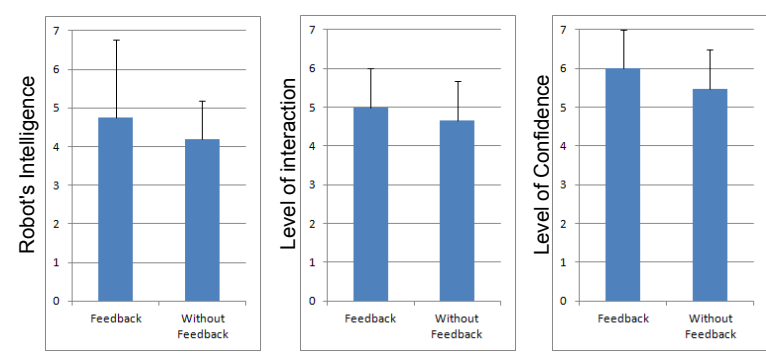

Fig. 19 Remote control. People's perception of the use of the remote control. Left: Robot's Intelligence. Center: Level of interaction. Right: Level of confidence.

mance of the robot approach proposed in the present paper.

\section{References}

Alami R, Albu-Schaeffer A, Bicchi A, Bischoff R, Chatila R, De Luca A, De Santis A, Giralt G, Guiochet J, Hirzinger G, et al (2006) Safe and dependable physical human-robot interaction in anthropic domains: State of the art and challenges. In: Proceedings of Workshop on pHRI, International conference on Intelligent Robots and Systems IROS, Citeseer, vol 6

Andrieu C, De Freitas N, Doucet A, Jordan MI (2003) An introduction to mcmc for machine learning. Machine learning 50(1-2):5-43

Arras K, Mozos O, Burgard W (2007) Using boosted features for the detection of people in $2 \mathrm{~d}$ range data. In: IEEE International Conference on Robotics and Automation, pp 3402-3407

Bennewitz M, Burgard W, Cielniak G, Thrun S (2005) Learning motion patterns of people for compliant robot motion. The International Journal of Robotics Research 24(1):31-48

Bergstrom N, Kanda T, Miyashita T, Ishiguro H, Hagita N (2008) Modeling of natural human-robot encounters. In: IEEE/RSJ International Conference on Intelligent Robots and Systems, pp 2623-2629

Burgard W, Cremers A, Fox D, Hähnel D, Lakemeyer G, Schulz D, Steiner W, Thrun S (1999) Experiences with an interactive museum tour-guide robot. Artificial Intelligence 114(1):3-55 
Dautenhahn K, Woods S, Kaouri C, Walters M, Koay K, Werry I (2005) What is a robot companion-friend, assistant or butler? In: IEEE/RSJ International Conference on Intelligent Robots and Systems, pp 11921197

Dautenhahn K, Walters M, Woods S, Koay K, Nehaniv C, Sisbot A, Alami R, Siméon T (2006) How may i serve you?: a robot companion approaching a seated person in a helping context. In: Proceedings of the 1st ACM SIGCHI/SIGART conference on Human-robot interaction, ACM, pp 172-179

Ferrer G, Sanfeliu A (2011) Comparative analysis of human motion trajectory prediction using minimum variance curvature. In: Proceedings of the 6th international conference on Human-robot interaction, ACM, Lausanne, Switzerland, pp 135-136

Ferrer G, Sanfeliu A (2014) Bayesian human motion intentionality prediction in urban environments. Pattern Recognition Letters 44:134-140, DOI http://dx.doi.org/10.1016/j.patrec.2013.08.013, URL http://www.sciencedirect.com/science/ article/pii/S0167865513003176

Foka A, Trahanias P (2010) Probabilistic autonomous robot navigation in dynamic environments with human motion prediction. International Journal of Social Robotics 2(1):79-94

Fong T, Nourbakhsh I, Dautenhahn K (2003) A survey of socially interactive robots. Robotics and autonomous systems 42(3):143-166

Garrell A, Sanfeliu A (2012) Cooperative social robots to accompany groups of people. The International Journal of Robotics Research 31(13):1675-1701

Goldberg D (1988) Genetic Algorithms in Search, Optimization \& Machine Learning. Addison-Wesley

Haasch A, Hohenner S, Hüwel S, Kleinehagenbrock M, Lang S, Toptsis I, Fink G, Fritsch J, Wrede B, Sagerer G (2004) Biron-the bielefeld robot companion. In: Proc. Int. Workshop on Advances in Service Robotics, Stuttgart, Germany: Fraunhofer IRB Verlag, pp 27-32

Hall ET (1969) The hidden dimension. Anchor Books New York

Helbing D, Molnár P (1995) Social force model for pedestrian dynamics. Physical review E 51(5):4282

Henry P, Vollmer C, Ferris B, Fox D (2010) Learning to navigate through crowded environments. In: Robotics and Automation (ICRA), 2010 IEEE International Conference on, IEEE, pp 981-986

Huang WH, Fajen BR, Fink JR, Warren WH (2006) Visual navigation and obstacle avoidance using a steering potential function. Robotics and Autonomous Systems 54(4):288-299
Ikeda T, Chigodo Y, Rea D, Zanlungo F, Shiomi M, Kanda T (2012) Modeling and prediction of pedestrian behavior based on the sub-goal concept. In: Robotics: Science and Systems

Ishiguro $\mathrm{H}$, Ono $\mathrm{T}$, Imai $\mathrm{M}$, Maeda $\mathrm{T}$, Kanda $\mathrm{T}$, Nakatsu R (2001) Robovie: an interactive humanoid robot. Industrial robot: An international journal 28(6):498-504

Katagami D, Yamada S (2003) Active teaching for an interactive learning robot. In: The 12th IEEE International Workshop on Robot and Human Interactive Communication, IEEE, pp 181-186

Khatib O (1985) Real-time obstacle avoidance for manipulators and mobile robots. In: IEEE Proc. of the international conference on Robotics and Automation, vol 2, pp 500-505

Koay K, Zivkovic Z, Krose B, Dautenhahn K, Walters M, Otero N, Alissandrakis A (2006a) Methodological issues of annotating vision sensor data using subjects' own judgement of comfort in a robot human following experiment. In: The 15th IEEE International Symposium on Robot and Human Interactive Communication, IEEE, pp 66-73

Koay K, Sisbot E, Syrdal D, Walters M, Dautenhahn K, Alami R (2007) Exploratory studies of a robot approaching a person in the context of handing over an object. Proc of AAAI-SS on Multi-disciplinary Collaboration for Socially Assistive Robotics pp 18-24

Koay KL, Dautenhahn K, Woods S, Walters ML (2006b) Empirical results from using a comfort level device in human-robot interaction studies. In: Proceedings of the 1st ACM SIGCHI/SIGART conference on Human-robot interaction, ACM, pp 194-201

Koren Y, Borenstein J (1991) Potential field methods and their inherent limitations for mobile robot navigation. In: IEEE International Conference on Robotics and Automation, IEEE, pp 1398-1404

Kuderer M, Kretzschmar H, Sprunk C, Burgard W (2012) Feature-based prediction of trajectories for socially compliant navigation. In: Proc. of Robotics: Science and Systems (RSS)

Luber M, Stork J, Tipaldi G, Arras K (2010) People tracking with human motion predictions from social forces. In: IEEE International Conference on Robotics and Automation, pp 464-469

Luber M, Tipaldi G, Arras K (2011a) Better models for people tracking. In: IEEE International Conference on Robotics and Automation, pp 854-859

Luber M, Tipaldi G, Arras K (2011b) Placedependent people tracking. The International Journal of Robotics Research 30(3):280-293

Madhava Krishna K, Alami R, Simeon T (2006) Safe proactive plans and their execution. Robotics and 
Autonomous Systems 54(3):244-255

Michalowski M, Sabanovic S, Simmons R (2006) A spatial model of engagement for a social robot. In: 9th IEEE International Workshop on Advanced Motion Control, pp 762-767

Nakauchi Y, Simmons R (2002) A social robot that stands in line. Autonomous Robots 12(3):313-324

Olivera V, Simmons R (2002) Implementing humanacceptable navigational behavior and a fuzzy controller for an autonomous robot. In: Proceedings WAF: 3rd Workshop on Physical Agents, Murcia, Spain, pp 113-120

Pacchierotti E, Christensen HI, Jensfelt P (2006) Embodied social interaction for service robots in hallway environments. In: Field and Service Robotics, Springer, pp 293-304

Pandey AK, Rachid A (2009) A step towards a sociable robot guide which monitors and adapts to the person's activities. In: IEEE International Conference on Advanced Robotics

Pransky J (2004) Social adjustments to a robotic future. Wolf and Mallett pp 137-59

Rios-Martinez J, Spalanzani A, Laugier C (2014) From Proxemics Theory to Socially-Aware Navigation: A Survey. International Journal of Social Robotics, $7(2), 137-15$

Sanfeliu A, Andrade-cetto J (2006) Ubiquitous Networking Robotics in Urban Settings. In: Proceedings of Workshop on Network Robot Systems. Toward Intelligent Robotic Systems Integrated with Environments, pp 10-13

Satake S, Kanda T, Glas D, Imai M, Ishiguro H, Hagita N (2009) How to approach humans?: strategies for social robots to initiate interaction. In: 4th ACM/IEEE International Conference on Human-Robot Interaction, pp 109-116

Shi D, Collins E, Donate A, Liu X, Goldiez B, Dunlap D (2008) Human-aware robot motion planning with velocity constraints. In: International Symposium on Collaborative Technologies and Systems, IEEE, pp 490-497

Sisbot E, Marin-Urias L, Alami R, Simeon T (2007) A human aware mobile robot motion planner. IEEE Transactions on Robotics 23(5):874-883

Stein P, Spalanzani A, Santos V, Laugier C (2014) Leader following: A study on classification and selection. Robotics and Autonomous Systems DOI 10.1016/j.robot.2014.09.028, URL https : //hal.inria.fr/hal-01073327

Svenstrup M, Bak T, Andersen HJ (2010) Trajectory planning for robots in dynamic human environments. In: IEEE/RSJ International Conference on Intelligent Robots and Systems
Tasaki T, Matsumoto S, Ohba H, Toda M, Komatani K, Ogata T, Okuno H (2004) Dynamic communication of humanoid robot with multiple people based on interaction distance. In: IEEE International Workshop on Robot and Human Interactive Communication, pp $71-76$

Thrun S, Burgard W, Fox D, et al (2005) Probabilistic robotics, vol 1. MIT press Cambridge, MA

Trulls E, Corominas Murtra A, Pérez-Ibarz J, Ferrer G, Vasquez D, Mirats-Tur J, Sanfeliu A (2011) Autonomous navigation for mobile service robots in urban pedestrian environments. Journal of Field Robotics

Vasquez D, Okal B, Arras KO (2014) Inverse Reinforcement Learning algorithms and features for robot navigation in crowds: An experimental comparison. In: IEEE/RSJ International Conference on Intelligent Robots and Systems

Walters M, Dautenhahn K, Woods S, Koay K (2007) Robotic etiquette: results from user studies involving a fetch and carry task. In: 2nd ACM/IEEE International Conference on Human-Robot Interaction, pp 317-324

Wilkes D, Pack R, Alford A, Kawamura K (1997) Hudl, a design philosophy for socially intelligent service robots. Socially Intelligent Agents pp 140-145

Zanlungo F, Ikeda T, Kanda T (2011) Social force model with explicit collision prediction. EPL (Europhysics Letters) 93(6):68,005

Ziebart BD, Ratliff N, Gallagher G, Mertz C, Peterson K, Bagnell JA, Hebert M, Dey AK, Srinivasa S (2009) Planning-based prediction for pedestrians. IEEE/RSJ International Conference on Intelligent Robots and Systems pp 3931-3936

Zinn M, Khatib O, Roth B, Salisbury J (2004) Playing it safe [human-friendly robots]. IEEE Robotics \& Automation Magazine 11(2):12-21 